\title{
Social Mental Health during COVID-19 Pandemic in Iran: A Cross-sectional Study among the General Population of Razavi Khorasan Province, Iran
}

\author{
Maliheh Dadgarmoghaddam ${ }^{1}$, Mona Najaf Najafi², Alireza Ebrahimi ${ }^{3}$, Ali Talaei ${ }^{4}$, Neshat Najaf Najafi $^{5}$ and Amir Ali \\ Moodi Ghalibaf ${ }^{5, *}$ \\ ${ }^{1}$ Associate Professor of Community Medicine, Community Medicine Department, Faculty of Medicine, Mashhad University of Medical Sciences, Mashhad, Iran \\ ${ }^{2}$ Assistant Professor of Community Medicine, Clinical Research Unit, Faculty of Medicine, Mashhad University of Medical Sciences, Mashhad, Iran \\ ${ }^{3} \mathrm{PhD}$ Student in Curriculum Planning, Faculty of Education and Psychology, Ferdowsi University, Mashhad, Iran \\ ${ }^{4}$ Psychiatry and Behavioral Sciences Research Center, Mashhad University of Medical Sciences, Mashhad, Iran \\ ${ }^{5}$ Student Research Committee, Faculty of Medicine, Mashhad University of Medical Sciences, Mashhad, Iran
}

* Corresponding author: AmirAli Moodi Ghalibaf, Student Research Committee, Faculty of Medicine, Mashhad University of Medical Sciences, Mashhad, Iran. Tel: +989357705368; Email: amiralimoodi1998@gmail.com

Received 2020 June 10; Revised 2020 September 16; Accepted 2020 November 06.

\begin{abstract}
Background: The potential impact of the novel coronavirus pandemic on social mental health has become a great concern, and the current situation can cause different mental disorders.

Objectives: According to the importance of mental health, this study was conducted to evaluate the mental health status of the general population of Iran in Razavi Khorasan Province, Iran.

Methods: An online survey was conducted and made available for the general population of Razavi Khorasan through the snowball sampling technique within a week. The demographic information, contact history with COVID-19, and physical symptoms history of COVID-19 were collected by the survey. To maintain the population's mental health status, the Depression, Anxiety, and Stress Scale-21, which was developed by Lovibond and Lovibond (1995), was used. To reduce the risk of bias, the online survey was supposed to be filled with a maximum of two members of a family. The data were analyzed in SPSS software (version 21) by calculating descriptive statistics and using the Chi-square test. The significance level was considered at the $\mathrm{p}$-value of $<0.05$ in all analyses.

Results: Finally, 461 responders from Razavi Khorasan Province were included in this study. In total, $41.8 \%$, 32.1\%, and $34.5 \%$ of the responders had depression symptoms, were in anxiety mood, and had stress symptoms, respectively. The ratio of men to women was approximately 1:2. Most participants were in the age range of 30-40 years, and the most frequent marital status was being single. Families with 3-5 members constituted $81.5 \%$ of the responders. Near one-third of the studied population had a bachelor's degree.

Conclusion: According to the results of our study, it is important to pay attention to the general population's mental health status during the COVID-19 pandemic and conduct protocols to prevent or reduce the risk of mental disorders that can be caused by the current situation

Keywords: Anxiety, COVID-19, Depression, Mental health, Stress
\end{abstract}

\section{Background}

Today, the novel coronavirus disease 2019 (COVID-19) has become a great concern all over the world. The disease primarily appeared in China in December 2019 and after a while, the World Health Organization (WHO) expressed a global concern about the COVID-19 pandemic (1). This outbreak is becoming larger every day and, these days, it has become much greater than the last severe acute respiratory syndrome (SARS) outbreak in 2003 (2). According to the WHO reports, there are more than 18 million infected cases and more than 600,000 deaths due to COVID-19 worldwide. Among these infected cases and deaths, unfortunately, more than 312,000 and 17,000 were Iranian people, respectively (3).

Coronavirus disease 2019, like SARS, is a betacorona virus that is guessed to have been transmitted from bats to humans, although the actual source of the virus is still unknown (4). Up to now, the results of previous studies have shown that human-tohuman transmission via respiratory droplets is the main way of getting infected and an infected person can create up 4 new cases of $\operatorname{COVID}-19(5,6)$. Although different signs and symptoms were reported for the COVID-19 during its appearance, the most common ones were fever, fatigue, cough, sore throat, dyspnea, nausea, diarrhea, and vomiting; it is important to note that its features are updated daily (7). There is still no strong evidence of immunity in a specific group. The findings of numerous studies have indicated that older men with comorbidities are more likely to get infected, and having more severe diseases leads to aggravated outcomes. Such complications as cardiac injury, brain injury, respiratory failure, and death have been observed in severe cases (7-9) .

After the COVID-19 outbreak in Iran, the government made some limitations, such as quarantine, to control the disease. Due to these restrictive policies, people stayed at home, social centers were closed, and numerous entertainment activities were stopped, which eventually led to a "desperate plea" (10). In addition to these conditions, the lack of masks and other protective equipment, the 
fear of getting infected, and the loss of loved ones made people worried, and it seems to have strongly affected their mental health status (11). The results of studies during similar previous outbreaks and pandemics in other countries have shown that such situations cause different psychiatric morbidities, including increasing self-blame, stress, anxiety, depression, and other severe psychiatric disorders. These studies in developed countries helped the governments to apply appropriate policies to control the outbreak with fewer psychiatric complications $(12,13)$.

Numerous studies have been conducted to investigate the effects of the COVID-19 pandemic on the general population's mental health all over the world. Dousti et al. stated that Iranian COVID-19 patients, hospitalized or non-hospitalized, highly demonstrated perceived stress (14). Another study conducted in Iran by Shahriarirad et al. determined a major mental health problem in the general population, which was depression and anxiety (15). Further studies are being conducted in other countries to find out whether the COVID-19 outbreak have any effects on general population's mental health. Wang et al. reported the high possibility of mental health problems among the Chinese in this era; however, they suggested further studies to look for these kinds of problems and disorders (16). Currently, there is little information about the Iranian population's psychiatric status, especially in Razavi Khorasan Province, Iran. Therefore, this lack of information can cause wrong policies from the government and increase the rate of mental disorders during the pandemic. In this regard, this study was designed to assess the mental and psychiatric status of the general population in Razavi Khorasan Province, which is one of the biggest and populated provinces in Iran.

\section{Objectives}

This study aimed to evaluate the current mental and psychiatric status for further probable interventions and suggest the right solutions and policies to the government.

\section{Methods}

\subsection{Study design}

An online survey was conducted for a crosssectional study to assess the public's psychological status during the COVID-19 pandemic among the Iranian population. A snowball strategy was utilized to collect the data of the Iranians living in Razavi Khorasan Province in May 2020 for a week. The survey, in Farsi, was posted on social media to be available to the public within a week. The reason for using an online survey was to minimize face-to-face interaction. The inclusion criteria were having minimum literacy and access to the internet and social networks. On the other hand, the participants who did not fill out the survey completely were excluded. To reduce the risk of bias, it was requested that a maximum of two persons in a family fill the survey. All procedures were in line with the Ethics Committee of the Mashhad University of Medical Sciences, Mashhad, Iran (no. IR.MUMS.REC.1399.117), and informed consent was obtained from all respondents .

\subsection{Survey development}

The conducted survey consisted of questions in several areas: (1) demographic data (including gender, age, marital status, educational status, family size, and being medical staff) and (2) whether the subject had been visited by a doctor in the past 2 weeks due to COVID-19 symptoms, (3) had been hospitalized in the past 2 weeks due to COVID-19 symptoms, (4) had been tested for COVID-19 in the last 2 weeks, (5) had been quarantined in the past 2 weeks due to COVID-19 symptoms, (6) had a history of mental disorders, (7) had a history of treatment due to mental disorders, (8) had a history of comorbidity, (9) had been in close contact with a COVID-19 patient, (10) had been in direct contact with a COVID-19 patient (11), had been in contact with a suspected person of having COVID-19, and (12) had a mental health status.

Mental health status was measured using the Depression, Anxiety, and Stress Scale (DASS-21). This scale was previously used in research related to the SARS and has been demonstrated to be a reliable and valid measure in assessing mental health status $(17,18)$. The Persian version of the DASS-21 questionnaire was approved valid and reliable (Cronbach alpha coefficients were reported to be $0.77,0.79,0.78$ for depression, anxiety, and stress subscales, respectively) in the Iranian population by Sahebi et al. (19). Specific items in DASS-21 define each subscale; regarding, items 3, 5, $10,13,16,17$, and 21 have formed the depression subscale. The total scores of the items in the depression subscale are divided into normal (0-9), mild (10-13), moderate (14-20), severe (21-27), and extremely severe $(+28)$. Items $2,4,7,9,15,19$, and 20 have formed the anxiety subscale, in which the total anxiety scores are divided into normal (0-7), mild (8-9), moderate (10-14), severe (15-19), and extremely severe $(+20)$. Stress is measured by items $1,6,8,11,12,14$, and 18, and the total scores of stress are divided into normal (0-14), mild (15-18), moderate (19-25), severe (26-33), and extremely severe $(33+)$.

\subsection{Statistical analysi}

The statistical analysis was conducted in SPSS software (version 21). Descriptive statistics were calculated for demographic characteristics. The mean and the standard deviation were calculated for each subscale of the DASS-21 scale, and the Chi-square test 
was used for comparing the frequency between different groups. All tests were two-tailed, and the pvalues of $<0.05$ were considered significant.

\section{Results}

This study was conducted on 461 people living in Razavi Khorasan Province. Among the participants, 310 $(67.2 \%)$ cases were women. Most of the participants were in the age range of $30-40$ years $(30.2 \%)$ and the majority of responders were single (61.4\%). The most common household size in the study was 3-5 people. Most of the samples had a bachelor's degree (34.7\%) and $313(67.9 \%)$ subjects were considered medical staff. The demographic characteristics of the participants are presented in Table 1.

According to Table 2, 4.8\% of people were visited by a doctor 2 weeks before answering questions due to the symptoms of COVID-19, 3.9\% were tested, $4.1 \%$ were quarantined due to the symptoms of the disease, and only $0.4 \%$ were hospitalized. It was revealed $23.9 \%$ of the participants had a history of mental disorders and $16.5 \%$ of the subjects were receiving medical treatment due to mental disorders. There was also a history of chronic diseases in $13.4 \%$ of the participants.

\begin{tabular}{|c|c|c|c|c|c|}
\hline \multirow{2}{*}{$\begin{array}{l}\text { Variables } \\
\text { Gender }\end{array}$} & \multicolumn{2}{|c|}{ Frequency (\%) } & \multirow{2}{*}{$\begin{array}{l}\text { Variables } \\
\text { Educational status }\end{array}$} & \multicolumn{2}{|c|}{ Frequency (\%) } \\
\hline & Male & $151(32.8)$ & & Primary school & $9(2)$ \\
\hline & Female & $310(67.2)$ & & Secondary school & $101(21.9)$ \\
\hline \multirow[t]{6}{*}{ Age } & $12-20$ & $51(11.1)$ & & $\mathrm{BSc}$ & $160(34.7)$ \\
\hline & $>20-30$ & $128(27.8)$ & & MSc & $59(12.8)$ \\
\hline & $>30-40$ & $139(30.2)$ & & $\mathrm{PhD}$ & $132(28.6)$ \\
\hline & $>40-50$ & $74(16.1)$ & Family members & 1 person & $5(1.1)$ \\
\hline & $>50-60$ & $48(10.4)$ & & 2 persons & $61(13.2)$ \\
\hline & $>60$ & $21(4.6)$ & & $3-5$ persons & $376(81.6)$ \\
\hline \multirow[t]{4}{*}{ Marital status } & Married & $171(37.1)$ & & $\geq 6$ persons & $19(4.1)$ \\
\hline & Single & $283(61.4)$ & Healthcare personnel & Yes & $313(67.9)$ \\
\hline & Divorced & $5(1.1)$ & & No & $148(32.1)$ \\
\hline & Widowed & $2(0.4)$ & & & \\
\hline
\end{tabular}

Table 2. Frequency of COVID-19 symptoms, history of contact with COVID-19 patients, mental disorders, and comorbidities (n=461)

\begin{tabular}{lcc}
\hline Items & $\begin{array}{c}\text { Frequency } \\
\text { n (\%) }\end{array}$ \\
\hline To be visited by a doctor in the past 2 weeks due to COVID-19 symptoms & $22(4.8)$ \\
To be hospitalized in the past 2 weeks due to COVID-19 symptoms & $2(0.4)$ \\
To be tested for COVID-19 in the last 2 weeks & $18(3.9)$ \\
To be quarantined in the past 2 weeks due to COVID-19 symptoms & $(4.1)$ \\
History of mental disorders & $110(23.9)$ \\
History of treatment due to mental disorders & $76(16.5)$ \\
History of comorbidity & $62(13.4)$ \\
Close contact with a COVID-19 patient & $62(13.4)$ \\
\hline Indirect contact with a COVID-19 patient & $109(23.6)$ \\
\hline Contact with a suspected person of having COVID-19 & $128(27.8)$ \\
\hline
\end{tabular}

Based on the DASS-21, the depression, anxiety, and stress symptoms among responders were obtained at 267 (41.8\%), 205 (32.1\%), and 220 (34.5\%), respectively, due to the COVID-19. The results of the DASS-21, based on their intensity, are shown in figures 1,2 , and 3 .

According to the statistical analysis, there were significant differences between the two genders in their depression level $(44.7 \%$ in women, $36.3 \%$ in men, $\mathrm{P}=0.042$ ). Although there were more women with anxiety and stress disorders, no significant differences were observed in these terms between men and women (anxiety: 34.5\% in women, $27.4 \%$ in men, $\mathrm{P}=0.071$, stress: $37.1 \%$ in women, $29.3 \%$ in men, $\mathrm{P}=0.05)$. There was no statistically significant difference among people with possible depression and without depression in terms of age $(\mathrm{P}=0.461)$, marital status $(\mathrm{P}=0.655)$, educational status $(\mathrm{P}=0.339)$, family size $(\mathrm{P}=0.37)$, and being a medical staff $(\mathrm{P}=0.129)$.

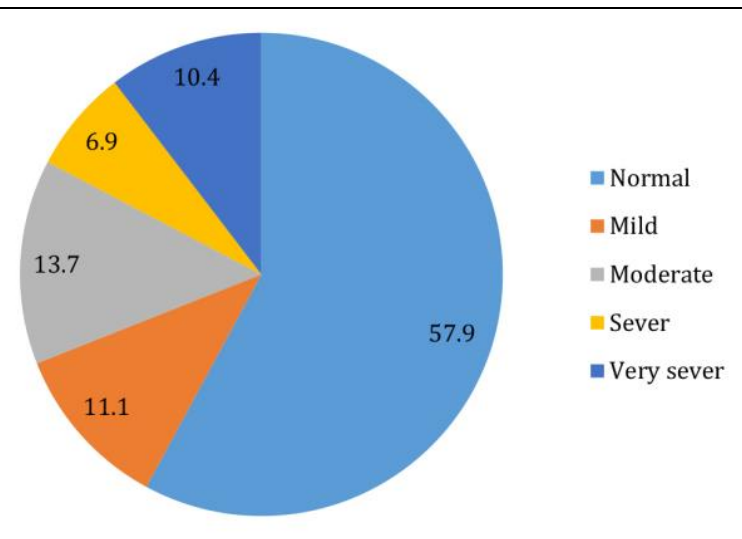

Figure 1. Percentage of the relative frequency of the survey respondents based on the severity of depression

The anxiety status in the cases with a possible anxiety disorder and without it had a statically 


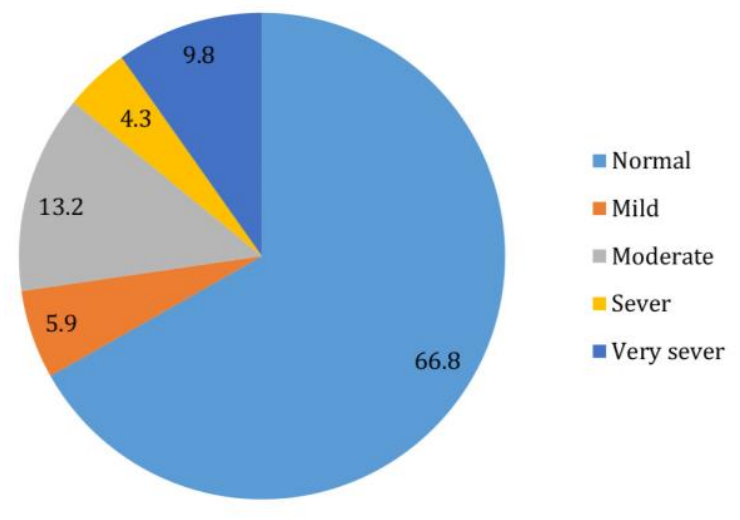

Figure 2. Percentage of the relative frequency of the survey respondents based on the severity of anxiety

significant relationship with age $(\mathrm{P}=0.026$; higher frequency in the age group of 30-50 years), marital status $(36 \%$ of the married participants were anxious, compared to $26.2 \%$ of the single ones, $\mathrm{P}=0.039$ ), and family size (cases with bigger family size had less anxiousness, $\mathrm{P}=0.036$ ). However, this relationship was not significant with educational status $(\mathrm{P}=0.058)$ and being a medical staff $(\mathrm{P}=0.184)$.

According to the results of our study, $37.7 \%$ of the medical staff and $28.9 \%$ of the non-medical staff had stress disorders, and the statistical analysis showed significant differences between these two groups $(\mathrm{P}=0.026)$. On the other hand, age $(\mathrm{P}=0.122)$, educational status $(\mathrm{P}=0.226)$, marital status $(\mathrm{P}=0.955)$, and family size $(\mathrm{P}=0.526)$ were not associated with stress.

It was found that the variable of staying at home and being quarantined was not associated with depression $(\mathrm{P}=0.824)$, anxiety $(\mathrm{P}=0.989)$, and stress $(\mathrm{P}=0.261)$. Moreover, comorbidities had no significant relationship with depressive disorder $(\mathrm{P}=0.189)$, anxiety disorder $(\mathrm{P}=0.124)$, and stress disorder $(\mathrm{P}=0.071)$.

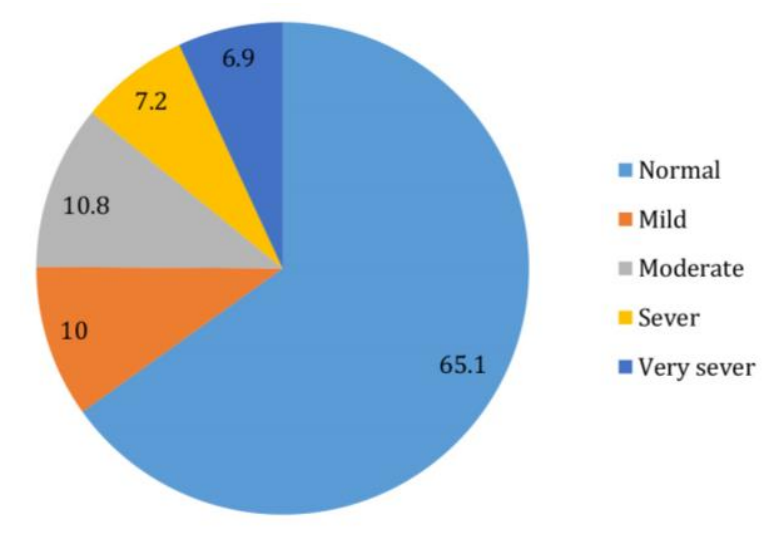

Figure 3. Percentage of the relative frequency of the survey respondents based on the severity of stress

\section{Discussion}

According to the findings of the current study, most of the cases' jobs were related to the medical environment and the medical team ( 68\%), and women's population was about as twice of men. In this study, the majority of responders were middle-aged, married, educated, and their family members were 3-5 people. The responses showed that nearly all the participants did not observe the quarantine during the COVID-19 pandemic, and the percentage of the people who visited a doctor, tested for infection, and were hospitalized were too small. Most of the responders were determined to have a good or excellent health status and approximately $15 \%$ of them had reported a history of comorbidities. More than three-quarters of the responders stated they lacked any direct and/or indirect contact with suspected and/or confirmed COVID-19 cases. The number of people with previous mental health disorders was interestingly high in this study. (\%24 )

In this study, the DASS-21 was analyzed in three subscales, namely stress, depression, and anxiety, according to which, more than half of the studied cases had normal status in each subscale $(\sim 65 \%, 58 \%$, and $67 \%$, respectively). Based on the statistical analysis, depression and stress had a significant relationship with being a medical staff; however, none of the subscales had a significant relationship with gender and age. Marital status showed a significant relationship with the depression score. The analysis of this study indicated a reverse relationship between the number of family members and each subscale; in this respect, more family members could be a protective factor against stress, depression, and anxiety. Similar to the number of family members, educational status had a reverse relation with every three subscales; therefore, higher education could protect them against stress, depression, and anxiety; however, higher education could be effective on the socio-economic status of the people that might affect the stress, depression, and anxiety strongly .

According to the findings of this study, people who visited a doctor had significantly lower anxiety levels; nevertheless, their stress and depression status showed no significant relationship in this regard. As the statistical analysis showed, there was no significant relationship of studied subscales with observing the quarantine and having comorbidities; nonetheless, it seems that comorbidities could be an effective factor in anxiety due to the calculated p-value. It was revealed that only the direct contact and depression had a significant relationship, and there was not any significant relationship between direct and/or indirect contact and other subscales, possibly indicating that individuals who were in contact with infected ones did not show any signs or symptoms to become stressed or anxious.

Regarding the importance of the mental health 
context, similar studies were conducted in other countries to investigate the population's mental health status and obtain an exact view of the needed interventions. The results of a study conducted by Wang et al. in China on 1,210 individuals showed a worrying status of stress, depression, and anxiety, highlighting the necessity of administrating effective interventions to improve the psychological status of the Chinese (20). Etxebarria et al. carried out a similar study in Spain, in which they investigated 976 adults. They mentioned that younger individuals with chronic diseases were reported to have more symptoms of psychological disorders and the low level of mental health impact at the start of the alerts decreased after stay-at-home order. Nevertheless, the findings of the mentioned study showed a higher level of mental and psychological problems after quarantine; however, the stay-at-home strategy seemed necessary for controlling the pandemic (21). The results of another study conducted by Samadarshi et al. in Nepal at the initial stage of the outbreak showed a high rate of stress in the Nepali population, and a strong association was observed between age and employment status (22).

Similar studies were performed in Latin countries rendering similar results. In Mexico, the poor psychological impact due to the COVID-19 outbreak was evaluated. Garcia-Priego et al. observed the high rates of stress, depression, and anxiety, and reported that younger age and internet addiction were the related factors with anxiety and depression among Mexicans (23). In the Middle East, the findings of a study conducted by Alyami et al. in Saudi Arabia showed a very low level of anxiety and depression, compared to those reported in the other mentioned studies. In the mentioned study, depression was strongly correlated with being elderly, having gotten divorced, being retarded, and being a university student. The married persons and those with higher incomes were associated with a higher risk of anxiety (24).

Due to the importance of the general population's mental health, having an exact and accurate overview of the current situation is essential for adopting the next steps, such as interventions. Here, some suggestions can be helpful for future studies and provide a guideline. As the first step of providing guidelines and making interventions is having an exact overview of the current situation, further studies need to be performed with a larger sample size. In this study, our studied population was limited to the Razavi Khorasan Province, and since our survey was conducted online, numerous people were excluded from the study due to lacking access to the Internet; therefore, greater studies in Iran and other provinces are suggested. Second, it can be helpful to use other methods, questionnaires, and scales to investigate the general population's mental health status in other studies. Third, attention to the studies conducted in other countries and their policies and strategies against this phenomenon can be beneficial for providing guidelines for the Iranian population. Fourth, paying attention to the vulnerable groups is important for authorities in any intervention and policy. Finally, it should not be forgotten that mental and psychological health can be effective on physical health; consequently, this subject intensifies the importance of mental health.

The limitations of the present study included an internet-based survey, its availability for a week among the population, and restricting the population to Razavi Khorasan Province. Nevertheless, our study had an acceptable number of survey responders of different strata of the population, the questions supported a wide range of people's characteristics, and the applied questionnaire was valid and reliable for the current pandemic situation.

\section{Conclusion}

According to the results of our study and other discussed studies, people's mental health is in danger during the COVID-19 pandemic and similar situations; regarding this, it seems important to have appropriate guidelines and protocols to prevent mental disorders caused by this condition. However, further studies are needed to have an exact view of mental health status in Iran.

\section{Footnotes}

Conflicts of Interest: The authors declare that they have no known competing financial interests or personal relationships that could have appeared to influence the work reported in this paper.

Financial Disclosure: The study was funded by Mashhad University of Medical Sciences, Mashhad, Iran.

\section{References}

1. Wang C, Horby PW, Hayden FG, Gao GF. A novel coronavirus outbreak of global health concern. Lancet. 2020;395(10223): 470-3. doi: 10.1016/S0140-6736(20)30185-9. [Pub Med: 31986257].

2. Hawryluck L, Gold WL, Robinson S, Pogorski S, Galea S, Styra R, et al. SARS control and psychological effects of quarantine, Toronto, Canada. Emerg Infect Dis. 2004;10(7):1206-12. doi: 10.3201/eid1007.030703. [Pub Med: 15324539].

3. Organization WH. Coronavirus disease 2019 ( COVID-19). situation report. 2020; 88.

4. Paules CI, Marston HD, Fauci AS. Coronavirus infectionsmore than just the common cold. Jama. 2020;323(8):707-8. doi: 10.1001/jama.2020.0757. [Pub Med: 31971553].

5. Huang C, Wang Y, Li X, Ren L, Zhao J, Hu Y, et al. Clinical features of patients infected with 2019 novel coronavirus in Wuhan, China. lancet. 2020;395(10223):497-506. doi :10.1016/S0140-6736(20)30183-5. [Pub Med: 31986264].

6. Cao Z, Zhang Q, Lu X, Pfeiffer D, Jia Z, Song H, et al. Estimating the effective reproduction number of the 2019-nCoV in China. MedRxiv. 2020. doi: 10.1101/2020.01.27.20018952.

7. Chen N, Zhou M, Dong X, Qu J, Gong F, Han Y, et al. Epidemiological and clinical characteristics of 99 cases of 2019 
novel coronavirus pneumonia in Wuhan, China: a descriptive study. Lancet. 2020;395(10223):507-13. doi: 10.1016/S01406736(20)30211-7. [Pub Med: 32007143].

8. Wu Y, Xu X, Chen Z, Duan J, Hashimoto K, Yang L, et al. Nervous system involvement after infection with COVID-19 and other coronaviruses. Brain Behav Immun. 2020;87:18-22. doi: 10.1016/j.bbi.2020.03.031. [Pub Med: 32240762].

9. Zhou F, Yu T, Du R, Fan G, Liu Y, Liu Z, et al. Clinical course and risk factors for mortality of adult inpatients with COVID-19 in Wuhan, China: a retrospective cohort study. lancet. 2020; 395(10229):1054-62. doi: 10.1016/S0140-6736(20)30566-3. [Pub Med: 32171076].

10. Horton R. Offline: 2019-nCoV-“A desperate plea". Lancet. 2020;395(10222):400. doi: 10.1016/S0140-6736(20)302993. [Pub Med: 32035537].

11. Xiang Y-T, Yang Y, Li W, Zhang L, Zhang Q, Cheung T, et al. Timely mental health care for the 2019 novel coronavirus outbreak is urgently needed. Lancet Psychiatry. 2020; 7(3):228-9. doi: 10.1016/S2215-0366(20)30046-8. [Pub Med: 32032543].

12. Sim K, Chan YH, Chong PN, Chua HC, Soon SW. Psychosocial and coping responses within the community health care setting towards a national outbreak of an infectious disease. J Psychosom Res. 2010;68(2):195-202. doi: 10.1016/j.jpsychores.2009.04.004. [Pub Med: 20105703].

13. Leung G, Lam T, Ho L, Ho S, Chan B, Wong I, et al. The impact of community psychological responses on outbreak control for severe acute respiratory syndrome in Hong Kong. $J$ Epidemiol Community Health. 2003;57(11):857-63. doi: 10.1136/jech.57.11.857. [Pub Med: 14600110].

14. Dousti P, Hosseininia N, Dousti P, Dousti S. Comparison of Catastrophizing and Perceived Stress in Three Groups of COVID19 Patients Recovered. Int J Indian Psychol. 2021;9(2):543-49. doi: 10.25215.0902.056.

15. Shahriarirad R, Erfani A, Ranjbar K, Bazrafshan A, Mirahmadizadeh A. The mental health impact of COVID-19 outbreak: a Nationwide Survey in Iran. Int J Ment Health Syst. 2021;15(1):1-3. doi: 10.1186/s13033-021-00445-3. [Pub Med: 33640006].

16. Wang Y, Shi L, Que J, Lu Q, Liu L, Lu Z, Xu Y, Liu J, Sun Y, Meng $S$, Yuan $K$, et al. The impact of quarantine on mental health status among general population in China during the COVID-
19 pandemic. Mol Psychiatry. 2021;26(9):1-10. doi: 10.1038/s41380-021-01019-y.

17. Asghari moghaddam MA, Saed F, Dibajnia P, Zangeneh J. A preliminery validation of the depresion, anxiety and stress scales (dass) in non-clinical sample. Daneshvarraftar. 2008;1(31):2338.

18. McAlonan GM, Lee AM, Cheung V, Cheung C, Tsang KW, Sham $\mathrm{PC}$, et al. Immediate and sustained psychological impact of an emerging infectious disease outbreak on health care workers. Can J Psychiatry. 2007;52(4):241-7. doi: 10.1177/0706743 70705200406. [Pub Med: 17500305].

19. Sahebi A, Asghari MJ, Salari RS. Validation of depression anxiety and stress scale (DASS-21) for an Iranian population. Iran J Psychiatry Behav Sci. 2005;1(4):1-18.

20. Wang C, Pan R, Wan X, Tan Y, Xu L, Ho CS, et al. Immediate psychological responses and associated factors during the initial stage of the 2019 coronavirus disease (COVID-19) epidemic among the general population in China. International journal of environmental research and public health. 2020;17(5):1-25. doi: 10.3390/ijerph17051729. [Pub Med: 32155789].

21. Ozamiz-Etxebarria N, Dosil-Santamaria M, PicazaGorrochategui M, Idoiaga-Mondragon N. Stress, anxiety, and depression levels in the initial stage of the COVID-19 outbreak in a population sample in the northern Spain. Cad Saude Publica. 2020;36(4):1-9. doi: 10.1590/0102-311X00054020. [Pub Med: 32374806].

22. Samadarshi SCA, Sharma S, Bhatta J. An online survey of factors associated with self-perceived stress during the initial stage of the COVID-19 outbreak in Nepal. Ethiop J Health Dev. 2020;34(2):84-9.

23. Garcia-Priego BA, Triana-Romero A, Pinto-Galvez SM, DuranRamos C, Salas-Nolasco O, Reyes MM, et al. Anxiety, depression, attitudes, and internet addiction during the initial phase of the 2019 coronavirus disease (COVID-19) epidemic: A cross-sectional study in Mexico. medRxiv. 2020:1-27. doi: 10.1016/j.jadr.2021.100097.

24. Alyami HS, Naser AY, Dahmash EZ, Alyami MH, Al Meanazel OT, Al-Meanazel AT, et al. Depression and anxiety during 2019 coronavirus disease pandemic in Saudi Arabia: a cross-sectional study. medRxiv. 2020;75(7):1-15. doi: $10.1101 / 2020.05 .09 .20096677$ 